\title{
A new combination of nanoparticles mass diffusion flux and slip mechanism approaches with electrostatic forces in a natural convective cavity flow
}

\author{
M. Mahdavi, M. Sharifpur*, H. Ghodsinezhad, J.P. Meyer \\ ${ }^{*}$ Corresponding author \\ *Tel: +27 124202448 "Email: mohsen.sharifpur@up.ac.za
}

Department of Mechanical and Aeronautical Engineering, University of Pretoria,

Private Bag X20, Hatfield, 0028, Pretoria, South Africa.

\begin{abstract}
Understanding of the phenomena involved in the mixture of nanoparticles and fluid requires more investigation in terms of many aspects. Both diffusion process and slip mechanisms are studied here with a new approach applied in the governing equations in Mixture model. The new approach tested for laminar natural convective flow inside a cavity (with two differentially heated walls) by using ANSYS FLUENT 15.0 with the presence of Alumina and Zinc Oxide nanofluids. The new slip mechanism covers the effects of virtual mass, pressure gradient, lift, buoyancy, centrifugal, van der waals attraction and electrical double layer repulsion forces. All the slip mechanism and source terms in the governing equations are implemented as User Define Functions in ANSYS FLUENT 15.0. The numerical results provided good agreement with experiments performed in this study. Depending on the volume fraction, heat transfer may improve or deteriorate, as reported by others. The comparison indicates that the ability of the proposed method is mainly associated to the concentration distribution, and of course in the ranges of volume fraction studied here. It is also found that the diffusion fluxes change the concentration profile near the diabatic walls, while the slip mechanism will be dominant in adiabatic walls.
\end{abstract}

Keywords: cavity, laminar natural convection, Mixture model, mass diffusion, slip mechanism, User Define Functions

\section{Introduction}

Enhancement of heat transfer in nanofluid binary mixture has brought this field of research to the interests of many academics. The increase in thermo-physical properties of a nanofluid results in the main cause of changing in flow and thermal features [1-9]. Although, the negative and positive effects on the thermal conductivity and viscosity has always remained controversial. For instance, Azmi et al. [10] and Rea et al. [11] showed that the importance of 
heat transfer improvement can come with the downside of pressure drops. On the other hand, experimental results of nanofluid in natural or even mixed convective flow indicate some deterioration in heat transfer [12, 13]. Ni et al. [13] conducted experiments of Alumina nanofluid inside a cavity with uniform temperature at the top and constant heat flux at the bottom. The results showed decrease in heat transfer for even volume fraction less than $0.2 \%$. There are not many experimental study of nanofluid natural convection inside a cavity on the literature. Here some are presented: Moradi et al. [14] pointed out that the highest Nusselt number occurred at 0.2 vol.\% of Alumina nanofluid in a cavity. Li and Peterson [15] only observed deterioration for the same nanofluid higher than 0.5 vol.\%. Ho et al. [16] stated that Nusselt number increases up to $4 \%$ volume fraction in an enclosure. $20 \%$ Increase in heat transfer was observed by Srinivas and Srivastava [17] in low 0.02 vol.\%. The decrease of Nusselt number only reported for higher than 2 vol.\% by Nnanna [18]. Ho et al. [19], Hu et al. [20] and Chang et al. [21] reported the decrease of heat transfer due to presence of nanoparticles inside a rectangular cavity.

The most applicable numerical model for nanofluid can be multiphase Mixture approach which assumes both liquid and solid particles as a continuum medium. However, the main challenge comes up when the slip mechanism between base fluid and particles are needed to be included. The default slip velocity only consists of buoyancy and centrifugal forces which are mentioned as the fundamental causes of induced drag force [22-25]. Buongiorno [26], Hwang et al. [27] , Kuznetsov and Nield [28] and Sheikholeslami et al. [29] considered the mass flux induced by Brownian and thermophoretic diffusion were the main phenomena in nanofluids. In fact, these were assumed the essential reasons of concentration distribution and heat transfer in nanofluids with implementing them only in mass and energy equations. On the other hand, Pakravan and Yaghoubi [30] applied Brownian and thermophoretic mechanisms as a slip velocity in all the flow field equations. Ho et al. [16] used both buoyancy as slip mechanism and mass diffusion conception of nanofluid stemmed from concentration gradient and thermophoresis in equations. They reported that the slip velocity has noticeable effects on final numerical results and concentration gradient and thermophoresis become important for volume fraction above 2\%. Hayat et al. [31 and 32] reported the temperature enhancement by Brownian parameter in non-Newtonian nanofluid in the presence of magnetic field.

Literature review shows that there are no complete agreement on nanofluid flow and heat transfer features, neither experimentally nor modelling. Therefore, considering all the 
involved phenomena at the same time in the equations seems necessary. In this study, natural convective flow of nanofluid in a cavity with differentially vertical heated walls is investigated. A typical schematic of a cavity with essential thermal boundary condition is shown in figure 1. Solar cavity receiver is one of the application of cavity flow. A new combination of mass diffusion and slip mechanism are considered as: mass diffusion induced by concentration gradient and thermophoresis in volume fraction and energy equations, slip velocity raised by attractive van der waals and repulsion electrical double layer, pressure gradient, virtual mass, lift, buoyancy and centrifugal forces.

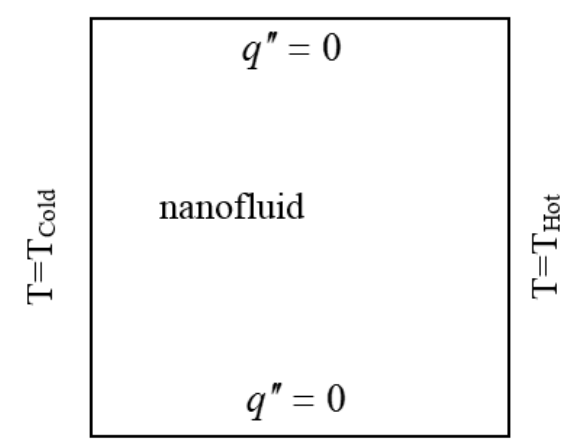

Figure 1. Problem description of a cavity in front view.

\section{Nomenclature}

\begin{tabular}{|c|c|c|c|}
\hline$A$ & Hamaker constant & $T_{f r}$ & reference temperature \\
\hline$C_{L}$ & lift coefficient & $V_{p m}, V_{k m}$ & drift velocity \\
\hline$c_{p}$ & specific heat & $V_{R}$ & potential energy \\
\hline$d$ & diameter & $V_{\text {slip }}$ & slip or relative velocity \\
\hline$d_{c}$ & water particle diameter & Special characters & \\
\hline$F$ & Faraday constant & $\phi$ & volume fraction \\
\hline$F_{V D W}$ & Van der waals force & $\beta$ & thermal expansion coefficient \\
\hline$F_{E D L}$ & repulsion force & $\varepsilon_{0}$ & vacuum permittivity \\
\hline$f_{d}$ & drag function & $\varepsilon_{r}$ & relative permittivity \\
\hline$\overline{I_{0}}$ & ionic strength & $\Gamma$ & mass source term \\
\hline$h$ & Particle to particle distance & $\kappa$ & Debye-Huckel parameter \\
\hline
\end{tabular}




\begin{tabular}{|c|c|c|c|}
\hline$J_{p}$ & Particle mass flux & $\lambda$ & mean free path \\
\hline$K_{B}$ & Boltzmann constant & $\mu$ & dynamic viscosity \\
\hline$K_{n}$ & Knudsen number & $v$ & Kinematic viscosity \\
\hline$k$ & Thermal conductivity & $\rho$ & density \\
\hline$L$ & cavity characteristic length & $\psi$ & surface potential \\
\hline$\dot{m}$ & flow rate & $\tau$ & particle relaxation time \\
\hline $\mathrm{Nu}$ & Nussult number & $\tau_{m}$ & mixture shear stress \\
\hline$P r$ & Prandtl number & Subscripts & \\
\hline$q$ & conduction heat flux & $c$ & Continues phase \\
\hline$R$ & gas universal constant & in & inlet \\
\hline$R a$ & Rayleigh number & $m$ & mixture \\
\hline$R e$ & Reynolds number & out & outlet \\
\hline$R e_{\omega}$ & vorticity Reynolds number & $p$ & particle \\
\hline $\bar{T}$ & temperature & & \\
\hline
\end{tabular}

\section{Experimental set-up}

Figure 2 illustrates the schematic and arrangement of experimental set-up used in this study. The size of the cavity is $96 \mathrm{~mm} \times 120 \mathrm{~mm}$ (toward $\mathrm{Y}$ and $\mathrm{Z}$ direction, respectively) and $102 \mathrm{~mm}$ (in $\mathrm{X}$ direction). Only two vertical walls were heated and cooled and the other walls are insulated. The constant temperature for hot and cold heat exchangers was supplied by two Polyscience thermostatic circulating baths (PD20R). The entire visible surfaces in figure $2 b$ were covered with insulator made of polystyrene and $20 \mathrm{~mm}$ in thickness. Three thermocouples with the accuracy of $\pm 0.02{ }^{\circ} \mathrm{C}$ are placed at the face of each hot and walls, as shown in Fig.1c. Seven thermocouples were placed at the mid-horizontal line of the cavity toward $\mathrm{X}$-axis from hot to cold wall and also 5 thermocouples at the mid-vertical line toward Y-axis from bottom to top of the horizontal insulated walls. More details about the experiment can be found in [33].

\section{Nanofluid preparation}

The nanoparticles used in this study are $\mathrm{Al}_{2} \mathrm{O}_{3}$ and $\mathrm{ZnO}$. These were provided from Nanostructure and Amorphous Material Inc, USA. $\mathrm{Al}_{2} \mathrm{O}_{3}$ nanoparticles with manufactured 
claimed diameter of $30 \mathrm{~nm}$ and $99.9 \%$ purity and $\mathrm{ZnO}$ with claimed diameter of $20 \mathrm{~nm}$. The other properties of both of the nanoparticles are presented in table 1 .

Required concentration of nanofluids is obtained by adding DI water supplied from Merck (pty) Ltd. Ultrasonic agitation probe (Qsonica Q-700 20KHz and $700 \mathrm{~W}$ with 5s pulse on 2s pulse off with amplitude (intensity) of 98\%) is used to break down aggregation of the nanoparticles. The size of nanoparticles were examined with transmission electron microscopy (TEM). Therefore, Zeta sizer ZS (Malvern instrument limited, UK) was used to measure mean particle size. The Zeta sizer is using 4mW He-Ne $633 \mathrm{~nm}$ LASER as a source. The stability of nanofluid was verified by direct visualization and also using Dynamic Light Scattering (DLS) method with Zeta sizer Nano ZS (Malvern ZS., UK). The measured zeta potential is above $25 \mathrm{mV}$ in all the cases. The measurement shows that the mean diameter of $\mathrm{Al}_{2} \mathrm{O}_{3}$ is $250 \mathrm{~nm}$ and equal to $127 \mathrm{~nm}$ for $\mathrm{ZnO}$. In this study, the reported particle diameter by manufacturers is not used in calculations and the measured one would be applicable. It must note that Zeta sizer gives the average of nanoparticles size in the nanofluid which it is usually different than the particles size as a powder. The volume fraction varies from $0.1 \%$ to $0.5 \%$ for $\mathrm{Al}_{2} \mathrm{O}_{3}$ and $0.1 \%$ to $1 \%$ for $\mathrm{ZnO}$.

Table 1. Thermo-physical properties of the nanoparticles

\begin{tabular}{lll}
\hline properties & $\mathrm{Al}_{2} \mathrm{O}_{3}$ & $\mathrm{ZnO}$ \\
\hline$\rho\left(k g / m^{3}\right)$ & 3950 & 5606 \\
$c_{p}(J / k g . K)$ & 765 & 523 \\
$k(W / m . K)$ & 36 & 25 \\
$d_{p}(n m)$ & 250 & 127
\end{tabular}

\section{Mathematical formulation}

\subsection{Mixture model equations}

Mixture model is one of the multiphase approaches with the assumption of continuum theory in highly coupled binary mixture. Checking the Knudsen number less than 0.1 proves the validity of the theory: for nanoparticles inside the liquid as which and are mean free path of the liquid phase $(0.3 \mathrm{~nm}$ for water), and nanoparticle mean diameter (normally from $50 \mathrm{~nm}$ to $300 \mathrm{~nm}$ ). The momentum and energy equations of both phases are simply combined to form a new set of equations. Particle flux diffusion due to disturbed concentration and thermophoresis are added to the right hand side of the mass and energy equation with the 
simple assumption of Fick's diffusion law. Also, the effects of slip and drift velocity in all the flow equation are included.

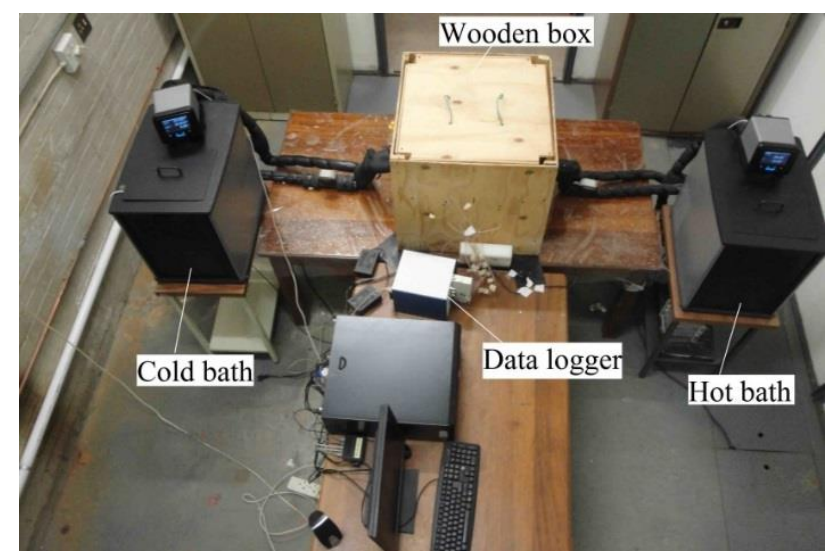

a)

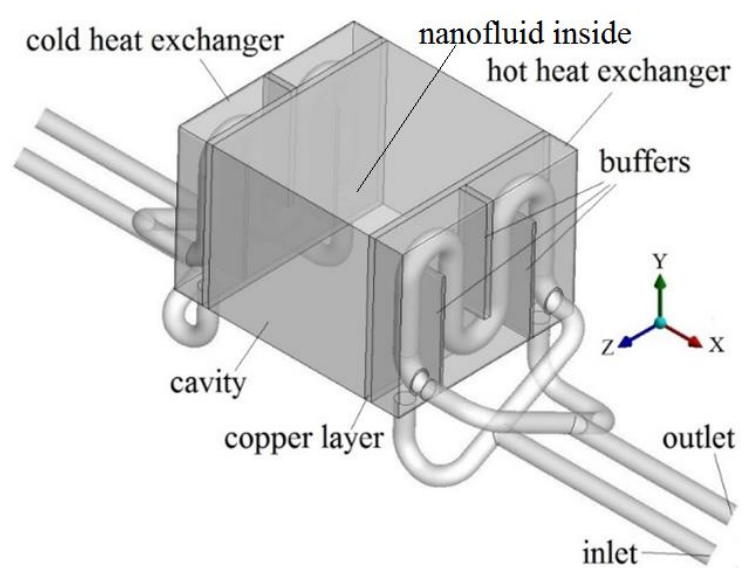

b)

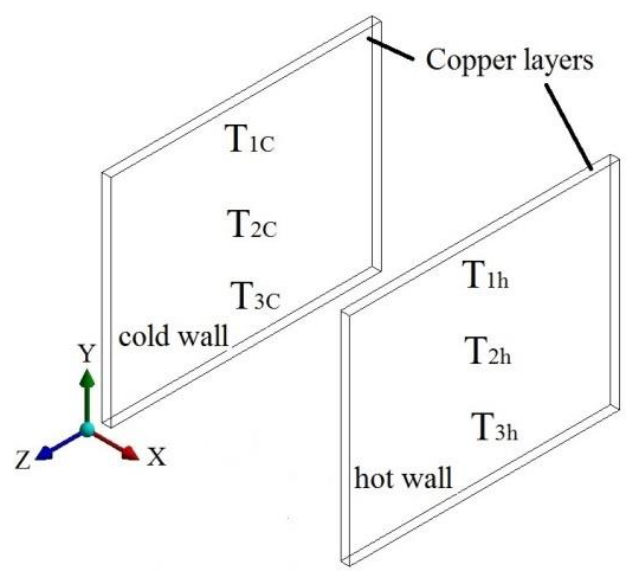

c)

Figure 2. a) Test section including heat exchangers and cavity b) schematic of cavity and heat exchangers c) Locations of thermocouples attached to the hot (h) and cold (c) walls The mass equation for each phase is [22]:

$\frac{\partial\left(\phi_{k} \rho_{k}\right)}{\partial t}+\nabla \cdot \phi_{k} \rho_{k} \vec{V}_{k}=\Gamma_{k}$

With the assumptions of steady flow and no mass transfer between phases, and linear summation of mass equation for both phases we reach:

$\nabla \cdot \sum_{k=1}^{2} \phi_{k} \rho_{k} \vec{V}_{k}=0$

It is noted that the net mass diffusion will be zero. Mixture continuity will be [22]:

$$
\nabla \cdot\left(\rho_{m} \vec{V}_{m}\right)=0
$$


$\vec{V}_{m}=\frac{\sum_{k=1}^{2} \phi_{k} \rho_{k} \vec{V}_{k}}{\rho_{m}}$

Mass equation for nanoparticles [26]:

$$
\begin{aligned}
& \nabla \cdot\left(\phi_{p} \rho_{p} \vec{V}_{m}\right)=-\nabla \cdot\left(\phi_{p} \rho_{p} \vec{V}_{p m}\right)-\nabla . J_{p} \\
& J_{p}=-\rho_{p} D_{B} \nabla \phi_{p}-\rho_{p} D_{T} \frac{\nabla T}{T} \\
& D_{B}=\frac{K_{B} T}{3 \pi \mu_{c} d_{p}} \\
& D_{T}=0.26 \frac{k_{c}}{k_{p}+2 k_{c}} \phi_{p} \frac{\mu_{c}}{\rho_{c}}
\end{aligned}
$$

where $J_{p}, \vec{V}_{p m}, D_{B}$ and $D_{T}$ are particle diffusion flux, drift velocity, mass diffusion coefficient and thermophoretic coefficient, respectively. $K_{B}$ is Boltzmann constant $1.38 \times 10^{-23}$. Then:

$$
\vec{V}_{p m}=\vec{V}_{p}-\vec{V}_{m}
$$

The drift and slip velocity are correlated as:

$$
\vec{V}_{p m}=V_{\text {slip }}-\frac{\phi_{p} \rho_{p}}{\rho_{m}} V_{s l i p}=\frac{\phi_{c} \rho_{c}}{\rho_{m}} V_{s l i p}
$$

The final form of the mass equation will be [26]:

$$
\nabla .\left(\phi_{p} \rho_{p} \vec{V}_{m}\right)=-\nabla .\left(\phi_{p} \rho_{p} \vec{V}_{p m}\right)+\rho_{p} D_{B} \nabla^{2} \phi+\rho_{p} D_{T} \frac{\nabla^{2} T}{T}
$$

A linear summation of momentum equation of each phase with together will reach to [22]:

$$
\begin{aligned}
\frac{\partial}{\partial t} \sum_{k=1}^{2}\left(\phi_{k} \rho_{k} \vec{V}_{k}\right)+\nabla \cdot \sum_{k=1}^{2}\left(\phi_{k} \rho_{k} \vec{V}_{k} \vec{V}_{k}\right) & =-\nabla \sum_{k=1}^{2}\left(\phi_{k} P_{k}\right) \\
& +\nabla \cdot \sum_{k=1}^{2}\left(\phi_{k} \tau_{k}\right)+\sum_{k=1}^{2} \phi_{k} \rho_{k} g
\end{aligned}
$$

The mixture momentum equation has to be re-arranged in terms of mixture variables and drift velocity [34]. 


$$
\begin{aligned}
\sum_{k=1}^{2} \phi_{k} \rho_{k} \vec{V}_{k} \vec{V}_{k} & =\sum_{k=1}^{2} \phi_{k} \rho_{k} \vec{V}_{k}\left(\vec{V}_{m}+\vec{V}_{k m}\right) \\
& =\rho_{m} \vec{V}_{m} \vec{V}_{m}+\sum_{k=1}^{2} \phi_{k} \rho_{k} \vec{V}_{k m} \vec{V}_{k m}
\end{aligned}
$$

$\vec{V}_{k m}$ is the drift velocity. Then, only the drift velocity of nanoparticles $\vec{V}_{p m}$ is needed to be substituted. Since we have $\vec{V}_{c m}=-\frac{\phi_{p} \rho_{p}}{\left(1-\phi_{p}\right) \rho_{c}} \vec{V}_{p m}$, with manipulation of above mentioned equations, the final form of momentum equation of the mixture will be $[34,35]$ :

$$
\nabla \cdot\left(\rho_{m} \vec{V}_{m} \vec{V}_{m}\right)=-\nabla P_{m}+\nabla \cdot \tau_{m}+\rho_{m} g-\nabla \cdot \frac{\phi_{p}}{\left(1-\phi_{p}\right)} \frac{\rho_{p} \rho_{m}}{\rho_{c}} \vec{V}_{p m} \vec{V}_{p m}
$$

Energy equation of the mixture [34]:

$$
\begin{aligned}
& \nabla \cdot\left(\rho_{m} C_{p_{m}} \vec{V}_{m} T_{m}\right)=-\nabla \cdot q_{m}-\nabla \cdot\left\lfloor\phi_{p} \rho_{p} \vec{V}_{p m} T_{m}\left(C_{p_{p}}-C_{p_{c}}\right)\right] \\
& +\rho_{p} C_{p_{p}}\left[D_{B} \nabla \phi \cdot \nabla T+D_{T} \frac{\nabla T \cdot \nabla T}{T}\right]
\end{aligned}
$$

where $C_{p_{m}}, C_{p_{p}}$ and $C_{p_{c}}$ are mixture, particle and fluid specific heat, respectively. More details about the derivation of the above mentioned equations can be found in [22, 26-28, 34$35]$.

\subsection{Parameters involved in the slip mechanism}

The force balance on a spherical particle in lagrangian frame is expressed as follows:

$$
F_{\text {inertia }}=F_{\text {drag }}+\sum F_{\text {others }}
$$

where $F_{\text {inertia }}, F_{\text {drag }}$ and $F_{\text {others }}$ are forces due to acceleration or virtual mass, drag and other forces including pressure gradient, lift, buoyancy, centrifugal, van der waals attraction and electrical double layer repulsion forces. Another arrangement of force balance is:

$$
F_{\text {drag }}=\sum F_{\text {others }}-F_{\text {inertia }}
$$

The drag force is directly related to the relative velocity between liquid and solid. Since the drag is equal to linear summation of all other interactions, each interaction can be virtually expressed as the cause of slip velocity in the equation. Therefore, a new form of the slip velocity can be arranged as follows: 
$V_{\text {slip }}=V_{\text {Virtual-mass }}+V_{\text {Pressure }}+V_{\text {lift }}+V_{\text {Buoyancy-centrifugal }}+V_{V D W}+V_{E D L}$

The right hand side are the slip velocity by virtual mass, pressure gradient, lift, buoyancy, centrifugal, van der waals attraction and electrical double layer repulsion forces, respectively.

Virtual mass and pressure gradient forces [22,36]:

$$
\begin{aligned}
& F_{\text {Virtual-mass }}=0.5 \frac{\rho_{c}}{\rho_{p}}\left(\vec{V}_{p} \nabla \vec{V}_{c}-\frac{d \vec{V}_{p}}{d t}\right) \\
& F_{\text {Pressure }}=\frac{\rho_{c}}{\rho_{p}} \vec{V}_{p} \nabla \vec{V}_{c}
\end{aligned}
$$

By comparing the slip mechanisms to the following equation, the final form of the slip velocity can be calculated [22]:

$$
\begin{aligned}
& F_{d r a g}=\frac{f_{d}}{\tau} \vec{V}_{\text {slip }} \\
& \tau=\frac{\rho_{p} d_{p}^{2}}{18 \mu_{c}}
\end{aligned}
$$

Where $\tau$ and $f_{d}$ are particle relaxation time and drag function based on particle Reynolds number as [37]:

$$
\begin{aligned}
& f_{d}=1+0.15 \operatorname{Re}_{p}^{0.687} \\
& \operatorname{Re}_{p}=\frac{d_{p} \rho_{c} V_{s l i p}}{\mu_{c}}
\end{aligned}
$$

Lift force [38, 39]:

$$
\begin{aligned}
& F_{\text {lift }}=C_{L} \frac{\rho_{c}}{\rho_{p}} \phi_{p}\left(\vec{V}_{p}-\vec{V}_{c}\right) \times\left(\nabla \times \vec{V}_{c}\right) \\
& C_{L}=6.46 \frac{3}{2 \pi \sqrt{\operatorname{Re}_{\omega}}} \\
& \operatorname{Re}_{\omega}=\frac{\rho_{c}\left|\nabla \times \vec{V}_{c}\right| d_{p}^{2}}{\mu_{c}}
\end{aligned}
$$

Slip velocity due to buoyancy and centrifugal forces [22]:

$$
F_{\text {Buoyancy-centrifugal }}=\frac{\left(\rho_{p}-\rho_{m}\right)}{\rho_{p}}\left(\vec{g}-\left(\vec{V}_{m} \cdot \nabla\right) \vec{V}_{m}-\frac{\partial \vec{V}_{m}}{\partial t}\right)
$$


Van der waals attractive force [36]:

$$
\begin{aligned}
& F_{V D W}=-\frac{6}{\pi d_{p}^{3}} \frac{A}{6 d_{p}} \frac{1}{x^{2}(x+1)^{3}(x+2)^{2}} \\
& x=\frac{h}{d_{p}}
\end{aligned}
$$

where $A$ and $h$ are Hamaker constant and surface to surface distance of two approaching particles. The amount of Hamaker constant is available for some common solid liquid mixtures [36], $4 \times 10^{-20} \mathrm{~J}$ in the case of Alumina nanofluid and $1.9 \times 10^{-20} \mathrm{~J}$ for Zinc oxid nanofluid.

Electrical double layer repulsion force [40]:

$$
\begin{array}{ll}
V_{R}=\pi d_{p} \varepsilon_{0} \varepsilon_{r} \psi^{2} \exp (-\kappa h) & \kappa d_{p}<10 \\
V_{R}=\pi d_{p} \varepsilon_{0} \varepsilon_{r} \psi^{2} \operatorname{Ln}[1-\exp (-\kappa h)] & \kappa d_{p}>10 \\
F_{E D L}=-\frac{6}{\pi d_{p}^{3}} \frac{d V_{R}}{d h} &
\end{array}
$$

where $V_{R}$ is the repulsion energy on the surface of a particle. The amount of vacuum and relative permittivity of the medium are $\varepsilon_{0}=8.854 \times 10^{-12} C V^{-1} m^{-1}$ and $\varepsilon_{r}=80$ for water, respectively. $\psi$ is the potential on the surface of the electrical double layer over charged surface group, which can be approximated by zeta potential on the surface of diffuse layer. Since the small zeta potential means less repulsion barriers and consequently producing stronger agglomeration inside the nanofluid, the approaching potential to zero is avoided in all the simulations. $\kappa$ is defined as Debye-Huckel parameter calculated from the following [40]:

$$
\begin{aligned}
& \kappa=\sqrt{\frac{2000 F^{2} I_{0}}{\varepsilon_{0} \varepsilon_{r} R T}} \\
& F=96485 \mathrm{Cmol}^{-1}, R=8.31 \mathrm{Jmol}^{-1} \mathrm{~K}^{-1}
\end{aligned}
$$

where $F$ and $R$ are Faraday and gas universal constant, respectively. The ionic strength is simply calculated by knowing the concentration $\left(\mathrm{c}_{\mathrm{i}}\right)$ and the charge $\left(\mathrm{z}_{\mathrm{i}}\right)$ of the species. With the assumption of the presence of only two ions $\left(\mathrm{H}^{+}\right.$and $\left.\mathrm{OH}^{-}\right)$in the mixture we have: 
$I_{0}=\frac{1}{2} \sum_{i} c_{i} z_{i}=\frac{1}{2} \begin{cases}10^{-(14-P H)} \quad P H>7 \\ 10^{-P H} \quad P H \leq 7\end{cases}$

The inverse amount of Debye-Huckel parameter can be scaled as Debye length or the thickness of the electrical double layer $L_{D}=\kappa^{-1} m$. This length is implemented in the program as a cut-off distance to avoid overlapping of diffuse layer of nanoparticles.

\subsection{Mixture properties and modelling considerations}

Instead of using conventional Boussinesq approximation, a density correlation for water was employed in all the equations [41]:

$$
\rho_{\mathrm{c}}=0.0000152322 \mathrm{~T}^{3}-0.0183891979 \mathrm{~T}^{2}+6.6564073561 \mathrm{~T}+243.4039040229
$$

The other thermo-physical and transport properties of the nanofluid mixture are as follows [42]:

$$
\begin{aligned}
& \rho_{m}=\sum_{k=1}^{2} \phi_{k} \rho_{k} \\
& C_{P_{m}}=\frac{\sum_{k=1}^{2} \phi_{k} \rho_{k} C_{P_{k}}}{\rho_{m}} \\
& \frac{k_{m}}{k_{c}}=1+4.4 \operatorname{Re}^{0.4} \operatorname{Pr}^{0.66}\left(\frac{T}{T_{f r}}\right)^{10}\left(\frac{k_{p}}{k_{c}}\right) \phi_{p}^{0.66} \\
& \operatorname{Re}=\frac{\rho_{c} u_{B} d}{\mu_{c}} \\
& u_{B}=\frac{2 K_{B} T}{\pi \mu_{c} d_{p}^{2}} \\
& \frac{\mu_{m}}{\mu_{c}}=\frac{1}{1-34.87\left(d_{p} / d_{c}\right)^{-0.3} \phi_{p}^{1.03}}
\end{aligned}
$$

where $T_{f r}$ and $d_{c}$ are water freezing temperature and molecule diameter as $0.3 \mathrm{~nm}$. The list of known and unknown parameters are presented in table 2. 
Table 2. Summary of known and unknown parameters in the equations

\begin{tabular}{ll}
\hline known parameters & unknown parameters \\
\hline nanoparticles properties from table 1: & $\begin{array}{l}\text { mixture } \\
\text { pressure }\end{array}$ \\
$\rho, c_{p}, k, d_{p}$ & $\vec{V}_{m}, T_{m}, P_{m}$ \\
Cavity dimension, $96 \mathrm{~mm} \times 120 \mathrm{~mm} \times 102 \mathrm{~mm}$ & nanoparticles concentration distribution \\
cold wall temperature from $18^{\circ} \mathrm{C}$ to $25^{\circ} \mathrm{C}$ & from Eq. (5) \\
hot wall temperature from $33^{\circ} \mathrm{C}$ to $55^{\circ} \mathrm{C}$ & diffusion flux $J_{p}$ \\
volume fraction, from $0.1 \%$ to $0.5 \%$ for $\mathrm{Al}_{2} \mathrm{O}_{3}$ and & \\
$0.1 \%$ to $1 \%$ for $\mathrm{ZnO}$ & slip velocity $V_{s l i p}$ \\
zeta potential, $30 \mathrm{mV}$ & drift velocity $\vec{V}_{p m}$ \\
diffusion and thermophoresis coefficient in Eqs. & \\
(7) and (8) & \\
mixture properties in Eqs. (37) to (43)
\end{tabular}

\subsection{Computational procedure}

ANSYS Fluent 15.0 was employed to simulate the nanofluid-filled cavity flow. The slip velocity presented in this study was implemented into Mixture model via User Defined Functions (UDF). The UDF was divided into five sections: adjust function to define gradient of concentration and other second order of derivatives, slip velocity function, mixture thermo-physical properties, mass source term and energy source term functions. At each iteration, the first and second order of the derivatives for concentration and temperature are calculated through adjust functions. For this matter, few user scalers and memories are employed to save those derivatives. Therefore, diffusion terms can be calculated afterward. Then, slip velocity function is launched to consider the effects of all slip mechanisms through equation (18). Eventually, the mixture properties are calculated via knowing the temperature and concentration from previous iteration. All these functions are employed to finally solve the mass, momentum and energy equations of mixture in equations (3), (11), (14) and (15). 
Temperature boundary conditions are applied to the vertical cold and hot walls of cavity. The other 4 walls are assumed insulated and the gradient of temperature perpendicular to the walls is zero. No-slip boundary condition is considered at all the walls.

\section{Results and discussion}

Calculation of uncertainty in this study in terms of percentage shows $3 \%$ to $5.5 \%$ for Rayleigh number and $3.5 \%$ to $8 \%$ for Nusselt number.

Measurements on the hot and cold walls for different tests show a distribution of temperature in $\mathrm{Y}$ direction. This scattering of temperature is used as thermal boundary condition on modelling the cavity. Moreover, the average temperature on the walls is appeared in definition of Rayleigh number and thermo-physical properties as $g \rho_{m} C_{p_{m}} \beta_{m}\left(T_{h}-T_{c}\right) L^{3} / v_{m} k_{m}$ . Nusselt number is simply defined as $q^{\prime \prime} L /\left(T_{h}-T_{c}\right) k_{m}$. All the properties here are calculated at mean temperature of hot and cold walls and mixture properties in section 4.3.

The robustness of the model proposed in this study is verified by Nusselt number from measurements in figure 3. The good agreement between experiment and modelling is observed. The absolute error between calculated Nusselt number and measurements is presented in Table 3. As can be seen, the Nusselt number drops with increase in concentration, but still higher than distilled water in the case of Alumina nanofluid. These drop and rise were also reported by other researches $[14,15,17]$ in this ranges of volume fraction. Moreover, Alumina nanofluid provided higher heat rate and in lower volume fraction. The measured temperature on the mid-vertical line of the cavity from bottom to top of insulated walls is compared to numerical results in figure 4. This full agreement between simulation and experiment can be the main criterion for further predictions, especially for concentration distribution.

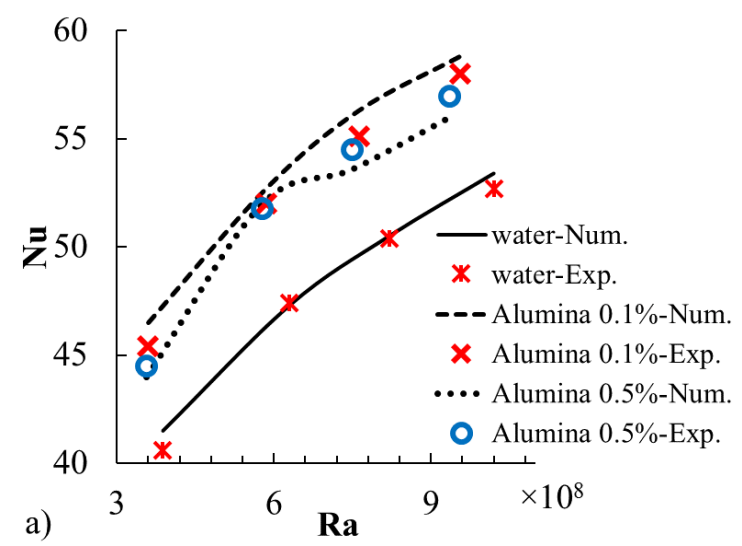




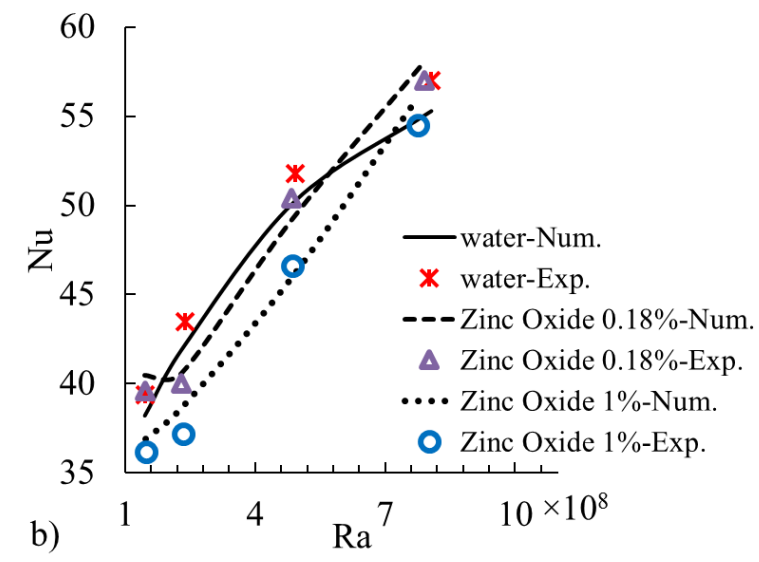

Figure 3. Comparison of Nusselt number measured during the experiments and calculated by numerical model for Alumina and Zinc Oxide nanofluids

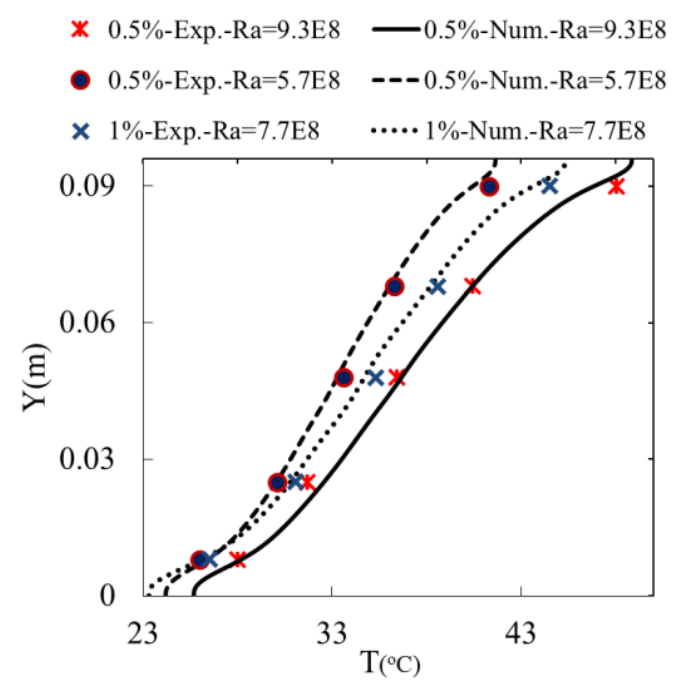

Figure 4. The profile of temperature in Y direction at the mid-vertical line of the cavity. All $0.5 \%$ vol. is for Alumina and only $1 \%$ vol. for Zinc Oxide nanofluid.

Table 3. Absolute error in calculation of Nusselt number comparing to experiment

\begin{tabular}{lll}
\hline nanofluid & volume fraction & Error in calculation of $\mathrm{Nu}(\%)$ \\
\hline Alumina & $0 \%$ & $0.2 \%$ to $3 \%$ \\
& $0.1 \%$ & $1 \%$ to $3 \%$ \\
& $0.5 \%$ & $0.4 \%$ to $2 \%$ \\
Zinc Oxide & $0 \%$ & $2 \%$ to $3 \%$ \\
& $0.18 \%$ & $1 \%$ to $3 \%$ \\
& $1 \%$ & $1 \%$ to $5 \%$ \\
\hline
\end{tabular}




\subsection{Prediction of flow, thermal and concentration distribution}

The most important capability of numerical simulation is prediction of flow features and concentration distribution of nanoparticles inside the domain. The strength of proposed combined method in this study is compared with the conventional one available in ANSYS Fluent 15.0 in figure 5.

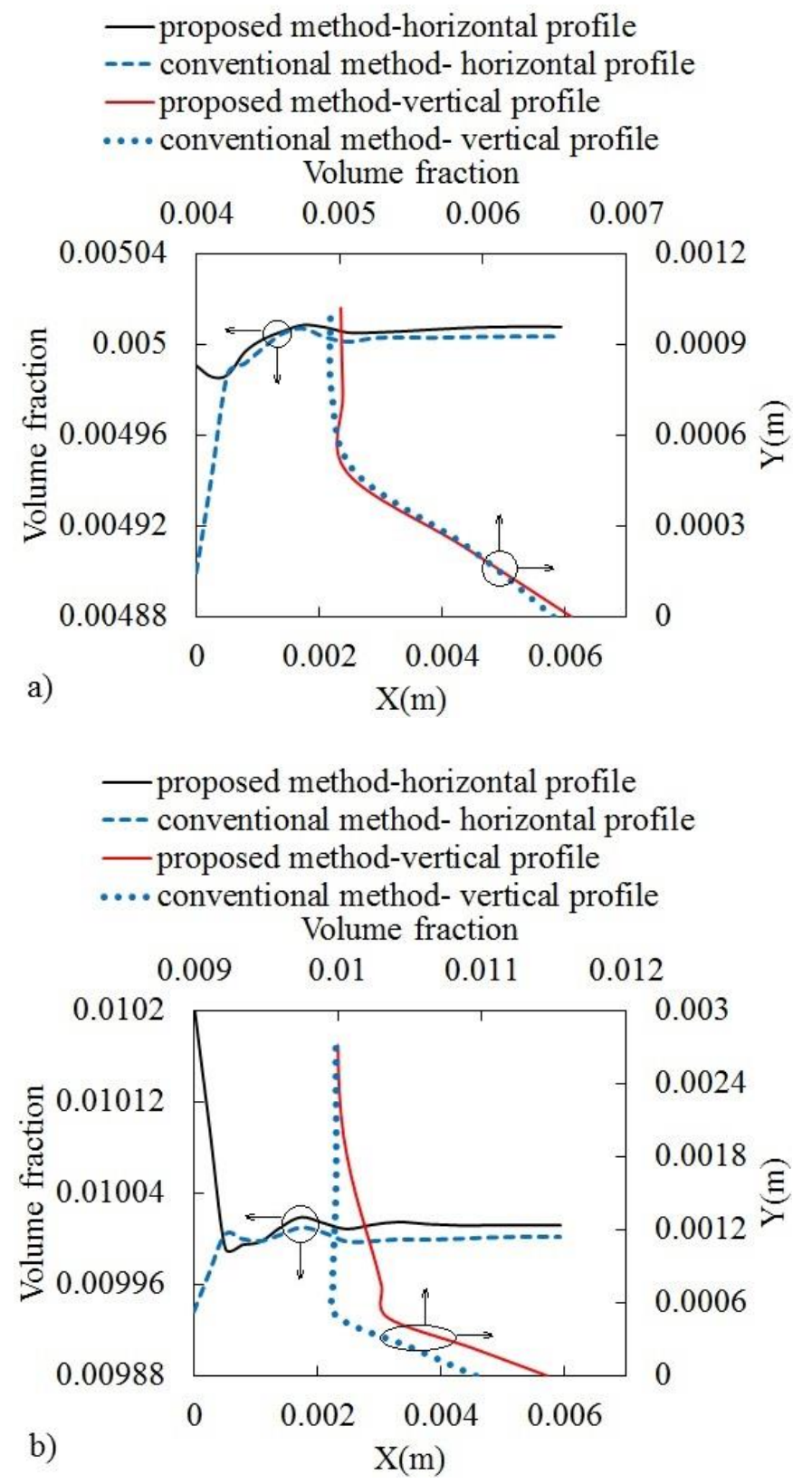

Figure 5. Concentration profile of a) Alumina $0.5 \%$ vol. $\mathrm{Ra}=9.3 \times 10^{8}$ b) Zinc Oxide $1 \%$ vol. $\mathrm{Ra}=7.7 \times 10^{8}$ nanoparticles at the vicinity of hot wall (horizontal) and from the bottom of the cavity (vertical) 
The conventional method in CFD software only includes the effects of gravitational force as the slip velocity and there are no terms for other slip mechanism and diffusion of nanoparticles. This provides wrong concentration distribution at the vicinity of the walls in figure 5. The impacts of other mechanisms are noticeable from $0.5 \%$ vol. for Alumina to $1 \%$ vol. for Zinc Oxide nanofluid. The higher amount of absolute value and gradient of volume fraction is clearly predicted close to the walls by the proposed method. It is caused by the presence of concentration and thermophoretic diffusion terms in mass equation, in particular next to the hot wall.

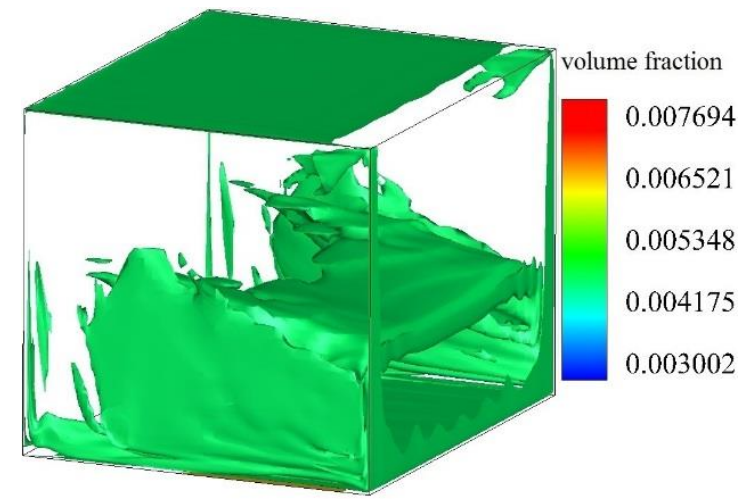

a) Alumina $0.5 \%$ vol. $\mathrm{Ra}=9.3 \times 10^{8}$, conventional method

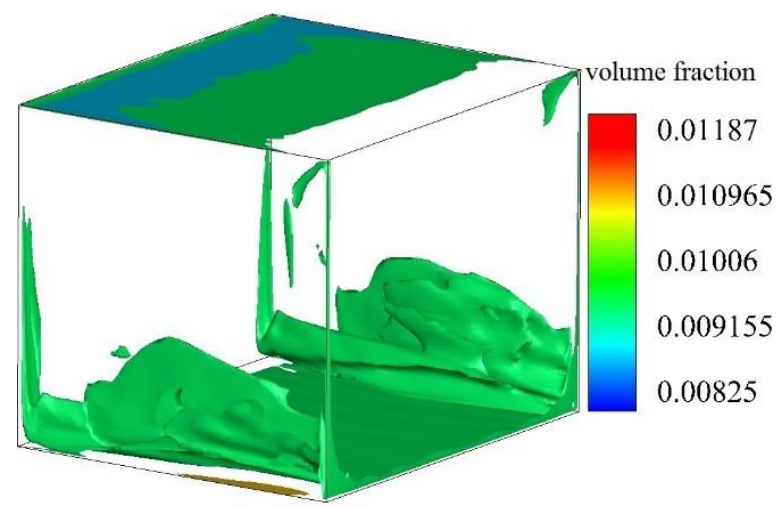

c) Zinc Oxide $1 \%$ vol. $\mathrm{Ra}=7.7 \times 10^{8}$, conventional method

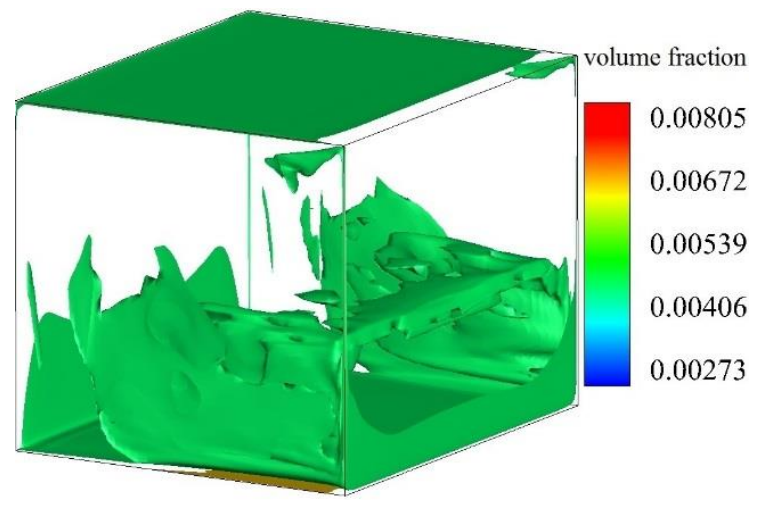

b) Alumina $0.5 \%$ vol. $\mathrm{Ra}=9.3 \times 10^{8}$, proposed method

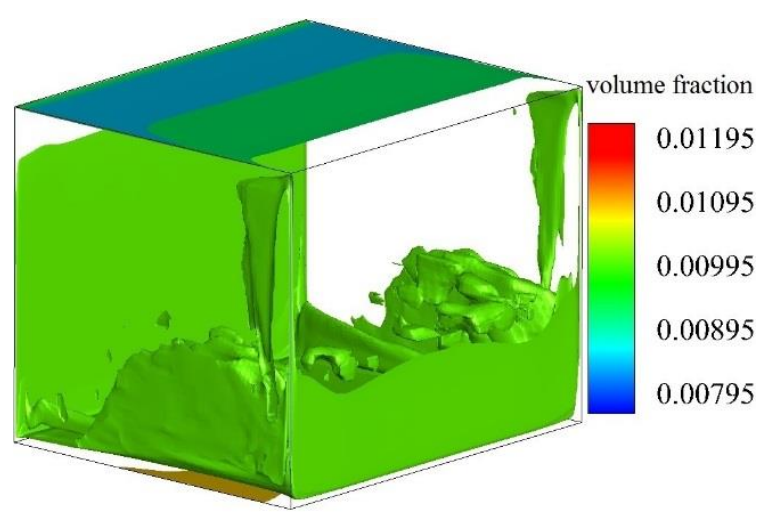

c) Zinc Oxide $1 \%$ vol. $\mathrm{Ra}=7.7 \times 10^{8}$, proposed method

Figure 6 . Three dimensional distribution of nanoparticles concentration in a cavity

One of the essential parts of prediction in this study is the concentration distribution of nanoparticles inside the cavity. It helps to have a deep understanding of particles migration along with the causes of changes in flow and thermal features, shown in figure 6 . The proposed method clearly presents different distribution than conventional method. The proposed method is able to predict the concentrated area at the bottom corners of the cavity and well-dispersion in other regions. 

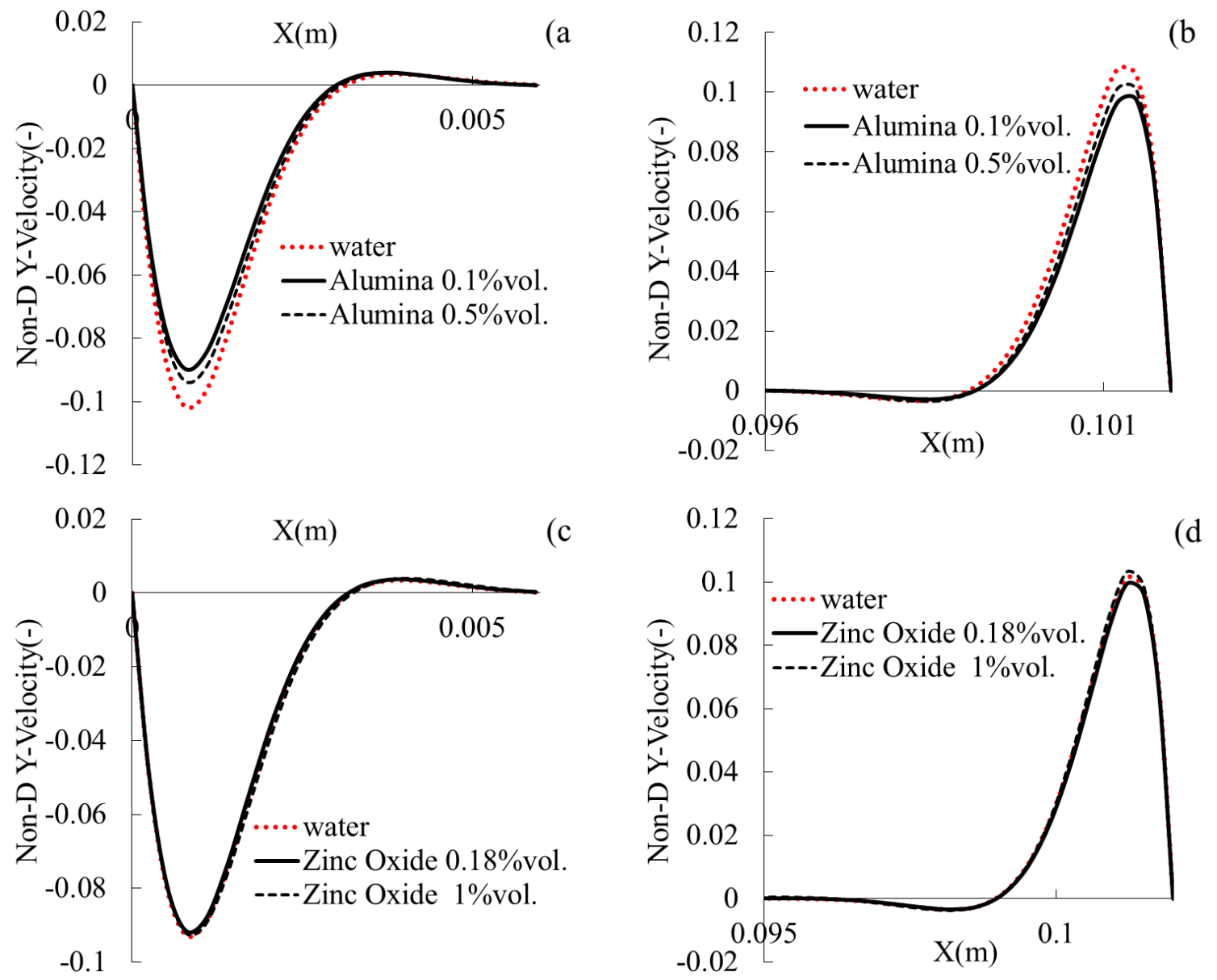

Figure 7. Non-dimensional Y velocity at the vicinity of the cold (first column) and hot (second column) walls on the mid-horizontal line from cold to hot walls

The profiles of the non-dimensional $\mathrm{Y}$ velocity $V / \sqrt{g \beta \Delta T L}$ for Alumina and Zinc Oxide nanofluid are compared to distilled water in Figure 7. The thickness of the hydrodynamic boundary layer is almost the same for all the cases. Since the concentration boundary layer of Zinc Oxide nanoparticles is thin close to the diabatic walls, shown in Figure 6, no effects of particles on velocity profile is observed in the case of Zinc Oxide nanofluid. It is mainly caused by buoyancy force on Zinc Oxide nanoparticles with higher density than Alumina particles, producing thicker concentration boundary layer at the bottom of the cavity in Figure 6. In the case of Alumina nanofluid, the competition among all the phenomena considered in this study will characterize the damping effects of particles on velocity. Therefore, the damping impacts on velocity in $0.5 \%$ vol can be less than $0.1 \%$ vol. 


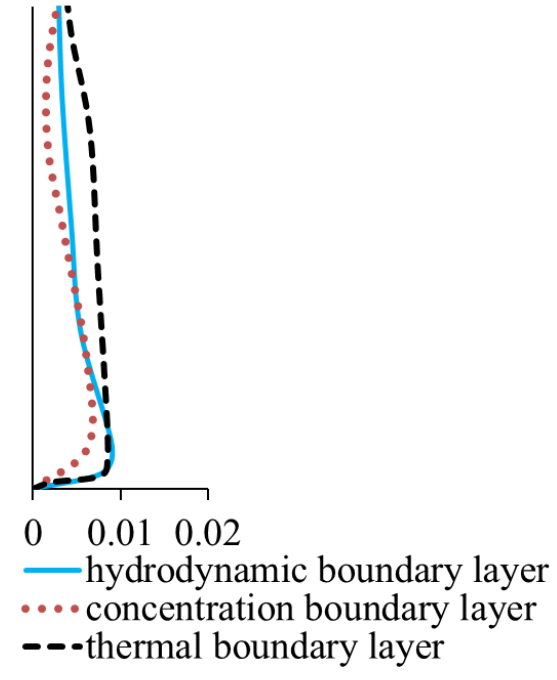

Alumina $0.5 \%$ vol., $\mathrm{Ra}=9.3 \times 10^{8}$

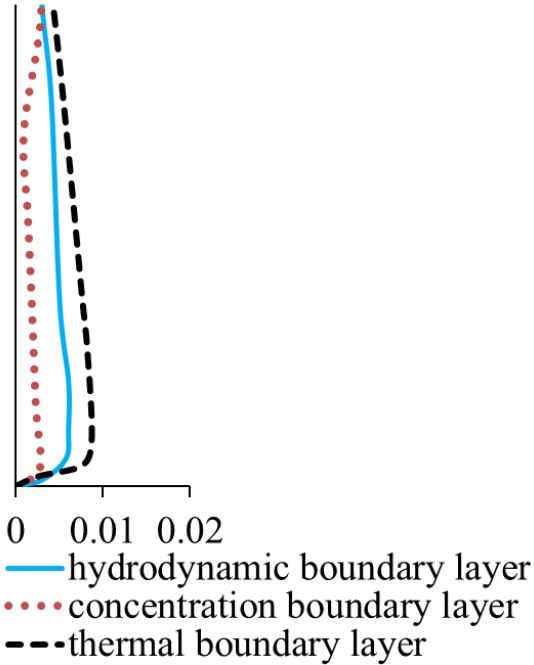

Zinc Oxide $1 \%$ vol. $\mathrm{Ra}=7.7 \times 10^{8}$

Figure 8. Flow, heat and mass boundary layers of nanofluids in natural convection close to the cold wall

The growth of hydrodynamic, concentration and thermal boundary layers could reveal many aspects of nanofluid flows, shown in Figure 8. The thermal boundary layer is the same for both nanofluids, and the thickest among others. The increase of nanoparticles density and volume fraction from Alumina to Zinc Oxide clearly affected the hydrodynamic boundary layer, particularly at the beginning of the growth. It is mainly caused by the presence of other slip mechanisms in momentum equation.

\section{Conclusion}

A new combination of mass diffusion conceptions and slip velocity of nanofluids in Mixture model was numerically investigated. The experimental setup consisted of laminar natural convection in a cavity with Alumina and Zinc Oxide nanofluids. The results of proposed method were found in good agreement with the experimental measurements, both Nusselt number and local measured temperature. As the volume fraction increased from $0.1 \%$ to $1 \%$, conventional Mixture model underestimated the concentration distribution, especially close to the walls and concentrated areas at the corners, comparing to the proposed one. Due to decrease in particle distances in higher volume fraction, the share of electrostatic forces increases in competition with bouncy force at the bottom of the cavity. Also, the presence of concentration and thermophoretic diffusion terms in mass equation thoroughly changed the nanoparticle concentration profile inside the boundary layer. Thermal boundary layer was found the thickest one among others (hydrodynamic and concentration boundary layers). The type of nanoparticles (mainly density effect) changed the hydrodynamic and concentration 
boundary layer. Concentration boundary layer reached its minimum thickness somewhere close to the middle of the diabatic wall, depending on the type of the nanoparticles. In summary, considering the diffusion and slip mechanism (particularly electrostatic forces) of nanoparticles should be the essential part of Mixture model for simulations of nanofluids, especially for higher volume fractions.

\section{References}

[1] C.H. Li, G.P. Peterson, Experimental investigation of temperature and volume fraction variations on the effective thermal conductivity of nanoparticle suspensions (nanofluids), Journal of applied physics, 99 (2006) 084314 .

[2] P.K. Namburua, D.P. Kulkarnia, D. Misrab, D.K. Das, Viscosity of copper oxide nanoparticles dispersed in ethylene glycol and water mixture, Experimental Thermal and Fluid Science, 32 (2007) 397-402.

[3] K. Nemade, S. Waghuley, A novel approach for enhancement of thermal conductivity of $\mathrm{CuO} / \mathrm{H}_{2} \mathrm{O}$ based nanofluids, Applied Thermal Engineering 95 (2016) 271-274.

[4] Y. Yang, Z.G. Zhang, E.A. Grulke, W.B. Anderson, Heat transfer properties of nanoparticle-in-fluid dispersions (nanofluids) in laminar flow, International Journal of Heat and Mass Transfer 48 (2005) 1107-1116.

[5] T. Srinivas, A.V. Vinod, Heat transfer intensification in a shell and helical coil heat exchanger using water-based nanofluids, Chemical Engineering and Processing 102 (2016) $1-8$.

[6] A.A. Minea, A study on Brinkman number variation on water based nanofluid heat transfer in partially heated tubes, Mechanics Research Communications 73 (2016) 7-11.

[7] S.Z. Heris, S.G. Etemad, M.N. Esfahany, Experimental investigation of oxide nanofluids laminar flow convective heat transfer, International Communications in Heat and Mass Transfer 33 (2006) 529-535.

[8] C. Liu, H. Lee, Y.H. Chang, S.P. Feng, The study of electrical conductivity and diffusion behavior of water-based and ferro/ferricyanide-electrolyte-based alumina nanofluids, Journal of Colloid and Interface Science 469 (2016) 17-24. 
[9] W. Williams, J. Buongiorno, L.W. Hu, Experimental Investigation of Turbulent Convective Heat Transfer and Pressure Loss of Alumina/Water and Zirconia/Water Nanoparticle Colloids (Nanofluids) in Horizontal Tubes Journal of heat transfer 130 (2008) 7.

[10] W.H. Azmi, K.V. Sharma, P.K. Sarma, R. Mamat, S. Anuar, V.D. Rao, Experimental determination of turbulent forced convection heat transfer and friction factor with $\mathrm{SiO}_{2}$ nanofluid, Experimental Thermal and Fluid Science 51 (2013) 103-111.

[11] U. Rea, T. McKrell, L. Hu, J. Buongiorno, Laminar convective heat transfer and viscous pressure loss of alumina--water and zirconia--water nanofluids. International Journal of Heat and Mass Transfer 52 (2009) 2042-2048.

[12] R. B. Mansour, N. Galanis, Experimental study of mixed convection with water-A12O3 nanofluid in inclined tube with uniform wall heat flux, International Journal of Thermal Sciences 50 (2011) 403-410.

[13] R. Ni, S.Q. Zhou, K.Q. Xia, An experimental investigation of turbulent thermal convection in water-based alumina nanofluid, Physics of fluid 23 (2011) 022005 .

[14] H. Moradi, B. Bazooyar, A. Moheb, S.G. Etemad, Optimization of natural convection heat transfer of Newtonian nanofluids in a cylindrical enclosure, Chinese Journal of Chemical Engineering 23 (2015) 1266-1274.

[15] C.H. Li, G.P. Peterson, Experimental Studies of Natural Convection Heat Transfer of A12O3/DIWater Nanoparticle Suspensions (Nanofluids), Advances in Mechanical Engineering 2 (2010) 742739.

[16] C.J. Ho, D.S Chen, W.M. Yan, O. Mahian, Buoyancy-driven flow of nanofluids in a cavity considering the Ludwig-Soret effect and sedimentation: Numerical study and experimental validation, International Journal of Heat and Mass Transfer 77 (2014) 684-694.

[17] S. Srinivas Rao, A. Srivastava, Interferometry-based whole field investigation of heat transfer characteristics of dilute nanofluids, International Journal of Heat and Mass Transfer 79 (2014) 166-175.

[18] A.G.A. Nnanna, Experimental Model of Temperature-Driven Nanofluid., Journal of Heat Transfer 129 (2007) 697-704. 
[19] C.J. Ho, W.K. Liu, Y.S. Chang, C.C. Lin, Natural convection heat transfer of aluminawater nanofluid in vertical square enclosures: An experimental study, International Journal of Thermal Sciences 49 (2010) 1345-1353.

[20] Y. Hu, Y. He, S. Wang, Q. Wang, H.I. Schlaberg, Experimental and Numerical Investigation on Natural Convection Heat Transfer of $\mathrm{TiO}_{2}-$ Water Nanofluids in a Square Enclosure, Journal of Heat Transfer 136 (2014) 022502.

[21] B.H. Chang, A.F. Mills, E. Hernandez, Natural convection of microparticle suspensions in thin enclosures, International Journal of Heat and Mass Transfer 51 (2008) 1332-1341.

[22] M. Manninen, V. Taivassalo, S. Kallio. On the mixture model for multiphase flow. Technical Research Centre of Finland: VTT Publications 288, 1996.

[23] M. Siavashi, H.R.T. Bahrami, H. Saffari, Numerical investigation of flow characteristics, heat transfer and entropy generation of nanofluid flow inside an annular pipe partially or completely filled with porous media using two-phase mixture model, Energy 93 (2015) 24512466.

[24] M. Mahdavi, M. Sharifpur, J.P. Meyer, CFD modelling of heat transfer and pressure drops for nanofluids through vertical tubes in laminar flow by Lagrangian and Eulerian approaches., International Journal of Heat and Mass Transfer 88 (2015) 803-813.

[25] F. Garoosi, B. Rohani, M.M. Rashidi, Two-phase mixture modeling of mixed convection of nanofluids in a square cavity with internal and external heating, Powder Technology 275 (2015) 304-321.

[26] J. Buongiorno, Convective transports in nanofluids., Journal of Heat Transfer, 128 (2006) 240-250.

[27] K.S. Hwang, S.P. Jang, S.U.S. Choi, Flow and convective heat transfer characteristics of water-based $\mathrm{Al}_{2} \mathrm{O}_{3}$ nanofluids in fully developed laminar flow regime, International Journal of Heat and Mass Transfer 52 (2009) 193-199.

[28] A.V. Kuznetsov, D.A. Nield, Natural convective boundary-layer flow of a nanofluid past a vertical plate, International Journal of Thermal Sciences 49 (2010) 243-247.

[29] M. Sheikholeslami, M. Gorji-Bandpy, S. Soleimani, Two phase simulation of nanofluid flow and heat transfer using heatline analysis, International Communications in Heat and Mass Transfer 47 (2013) 73-81. 
[30] H.A. Pakravan, M. Yaghoubi, Analysis of nanoparticles migration on natural convective heat transfer of nanofluids, International Journal of Thermal Sciences 68 (2013) 79-93.

[31] T. Hayat, M. Shafique, A. Tanveer, A. Alsaedi, Magnetohydrodynamic effects on peristaltic flow of hyperbolic tangent nanofluid with slip conditions and Joule heating in an inclined channel, International Journal of Heat and Mass Transfer 102 (2016) 54-63.

[32] T. Hayat, M. Shafique, A. Tanveer, A. Alsaedi, Hall and ion slip effects on peristaltic flow of Jeffrey nanofluid with Joule heating, Journal of Magnetism and Magnetic Materials 407 (2016) 51-59.

[33] M. Mahdavi, M. Sharifpur, H. Ghodsinezhad, J.P. Meyer, Boundary Condition Investigation for Cavity Flow Natural Convection. Kruger National Park, South Africa July 20-23, 11th International Conference on Heat Transfer, Fluid Mechanics and Thermodynamics (HEFAT 2015).

[34] M. Ishii, T. Hibiki. Thermo-Fluid Dynamics of Two-Phase Flow: Springer Science \& Business Media, 2006. chapter 13.

[35] T. Hibiki, M. Ishii, One-dimensional drift-flux model and constitutive equations for relative motion between phases in various two-phase flow regimes, International Journal of Heat and Mass Transfer 46 (2003) 4935-4948.

[36] J.S. Marshall, S. Li. Adhesive Particle Flow: A Discrete-Element Approach, Cambridge University, ISBN 1107032075, 9781107032071, 2014.

[37] Schiller, L., Naumann, A. A drag coefficient correlation. Zeitschrift Des Vereines Deutscher Ingenieure, 77 (1935) 318-320.

[38] D. A. Drew, R. T. Lahey. In Particulate Two-Phase Flow. Boston: ButterworthHeinemann, 1993 pp. 509-566.

[39] P.G. Saffman, The Lift on a Small Sphere in a Slow Shear Flow, Journal of fluid mechanics 22 (1965) 385-400.

[40] R.J. Hunter. Foundations of Colloid Science. Oxford University, 2001, pp. 599.

[41] D.G. Kröger, Air-cooled heat exchangers and cooling towers. PennWell Books, Appendix A, 2004. 
[42] M. Corcione, Empirical correlating equations for predicting the effective thermal conductivity and dynamic viscosity of nanofluids, Energy Conversion and Management 52 (2011) 789-793. 